\title{
VALERIANA MOYANOI SPEG. (VALERIANACEAE), NUEVO REGISTRO PARA LA FLORA DE CHILE
}

\author{
VALERIANA MOYANOI SPEG. (VALERIANACEAE), NEW RECORD FOR \\ THE CHILEAN FLORA
}

\author{
Patricio Saldivia ${ }^{1} \&$ Gloria Rojas ${ }^{2}$ \\ ${ }^{1}$ Laboratorio de Morfología y Taxonomía Vegetal, Facultad de Ciencias Agronómicas, Universidad de Chile. \\ patricio.saldivia.perez@gmail.com \\ ${ }^{2}$ Museo Nacional de Historia Natural, Casilla 787, Santiago, Chile.
}

\begin{abstract}
Valeriana moyanoi Speg. (Valerianaceae), known until today just for the Argentinean flora, was collected for the first time in Chile in two places of the XI Region of Aysén; Cerro Rosado and the Jeinimeni Lake National Reserve. This study includes a description of the species, its Chilean-Argentinean distribution, habitat and associate species.
\end{abstract}

La familia Valerianaceae comprende alrededor de 13 géneros y unas 300 especies, encontrándose en casi todo el mundo (Borsini et al. 1999). En Chile se encuentran cuatro géneros: Plectritis y Stangea con una especie, Valerianella con tres especies alóctonas (Matthei \& Espinoza 1997) y Valeriana, que hasta la fecha registraba 44 especies (Marticorena \& Quezada 1985; Marticorena 1990; Reese-Krug \& Weberling 1996). El género Valeriana comprende plantas hemicriptófitas, terófitas o sufrútices (caméfitos), con flores hermafroditas o unisexuales por aborto; cáliz entero, dentado o desarrollado en vilano; corola 5-mera, gibosa en la base; androceo reducido a 3 estambres fértiles, los dos restantes estériles o abortados; ovario ínfero; fruto aquenio. Se distribuyen especialmente en la Cordillera de los Andes (Borsini 1966).

La especie fue colectada en tres ocasiones: dos de ellas realizadas por Saldivia el 2 de enero del 2003 y el 2 de marzo del 2004 al Cerro Rosado, ubicado en la zona intermedia de la XI región, en la cuenca del río Emperador Guillermo (Fig. 1); y una realizada por Rojas y Saldivia en Enero del 2003 a la Reserva Nacional Lago Jeinimeni (Fig. 1), dentro del marco del proyecto "Flora de la Reserva Nacional Lago Jeinimeni” financiado por fondos patrimoniales de la DIBAM. En estas expediciones se co- lectaron individuos en estado vegetativo, en floración y en fructificación. Los ejemplares fueron herborizados y estudiados taxonómicamente (Borsini 1966; Borsini et al. 1999), y comparados con material colectado por Skottsberg en 1907 en Argentina de dos individuos en floración que se encuentran en el herbario del Museo Nacional de Historia Natural de Santiago (SGO), confirmando que se trata de la misma especie. En las revisiones bibliográficas, principalmente Marticorena \& Quezada (1985), y Henríquez et al. (1995), no se registró la presencia de Valeriana moyanoi para el territorio chileno. Los nuevos ejemplares de esta especie fueron depositados en SGO.

Material estudiado

ARGENTINA, Chubut, entre Lago Buenos Aires y Lago Ghio 1.700 m, 17-XII-1908, SKOTTSBERG (SGO 58789).

CHILE, XI Región, Prov. de Aysén, Comuna de Aysén, Cerro Rosado, 45 22'22S-7207’01W, 1.400 m, 02-I- 2003, SALDIVIA (SGO 152417) y 02- III2004, SALDIVIA (SGO 152416).

CHILE, XI Región, Prov. del General Carrera, Comuna de Chile Chico, Reserva Nacional Lago Jeinimeni, $46^{\circ} 50{ }^{\prime} 59 \mathrm{~S}-72^{\circ} 01^{\prime} 56 \mathrm{~W}, 1.600 \mathrm{~m}, 18$ - I2003, ROJAS \& SALDIVIA (SGO 152418). 


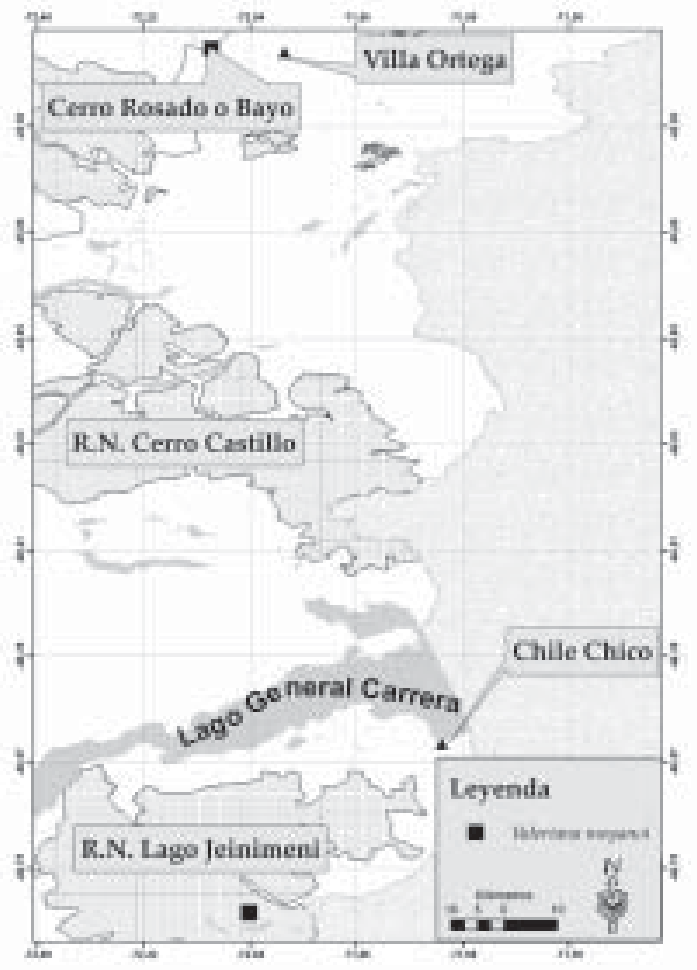

FIgURA 1. Localización de las colectas de Valeriana moyanoi en la región de Aysén.

FIGURE 1. Location of collections of Valeriana moyanoi in the Aysén Region.

DesCrIPCIÓn DE LA ESPECIE (Borsini et al. 1999), Fl. Patag. VI, pág. 462.

Valeriana moyanoi Speg. Revista Fac. Agron. Univ. Nac. La Plata 3: 607. 1897.

Hemicriptófito rosulado de hasta $20 \mathrm{~cm}$ de altura. Hojas radicales de hasta $6 \mathrm{~cm}$, densas, sésiles o pecioladas, lámina de 1 a $1,5 \mathrm{~cm}$, obovada, espatulada o lanceolada, carnosa, inciso-dentada o irregularmente lobada. Inflorescencias espiciformes frecuentemente densas. Flores perfectas de hasta 8 $\mathrm{mm}$ aproximadamente. Cáliz de $0,25 \mathrm{~mm}$. Corola de 3,5 a $6 \mathrm{~mm}$ anchamente infundibuliforme, gibosa en la base; tubo de $4 \mathrm{~mm}$, lóbulos de 1 a 1,5 mm, oblongos o anchamente ovados. Estambres de 1,5 $\mathrm{mm}$, anteras de 0,5 mm. Ovario de 1,2 mm. Estilo de 1,5 a $4 \mathrm{~mm}$, estigma trífido. Flores pistiladas de hasta 4,5 a $5 \mathrm{~mm}$. Cáliz de $0,5 \mathrm{~mm}$. Corola de 2 a 2,5 mm. campanulado-gibosa a 0,25 $\mathrm{mm}$ de la base aproximadamente, lóbulos ovados, obtusos. Ovario de 1 a $2 \mathrm{~mm}$. Estilo de 2,5 a $3 \mathrm{~mm}$, exerto y ensanchado hacia el ápice, estigma de $0,25 \mathrm{~mm}$, estaminodios de $0,5 \mathrm{~mm}$ (Fig. 2).

Los individuos colectados en Chile presentan una inflorescencia espiciforme muy densa, de entre 4,5 y $10 \mathrm{~cm}$ de largo; aquenios con una pubescencia muy leve, casi inadvertida en algunos casos, y además la base carnosa del vilano es de tan sólo 1 a $1,5 \mathrm{~mm}$.

Distribución: En Argentina se encuentra desde la provincia de Neuquén hasta la provincia de Santa Cruz (Borsini et al. 1999) y en Chile hasta la fecha se restringe únicamente a la XI Región de Aysén en la Reserva Nacional Lago Jeinimeni y en el Cerro Rosado (Fig.1).

HÁBitat: Entre piedras de la alta montaña por sobre el límite de bosque de la formación vegetacional Bosque caducifolio de Aysén (Gajardo 1994), entre los 1.000 hasta los $1.600 \mathrm{msnm}$ aproximadamente, donde por condiciones climáticas derivadas de la altitud se presenta una vegetación carente de árboles o arbustos altos (Pisano 1981).

ESPECIES ASOCIADAS: Se asocia a especies de formas de vida similares, adaptadas para vivir en este tipo de ambiente (Tabla I).

La presencia de $V$. moyanoi como un nuevo registro para la flora del territorio chileno, podría ser rebatida por la información entregada por Xifreda (1996), trabajo en el que además de mencionar las provincias argentinas en donde se encuentra la especie, se menciona a Chile como país limítrofe con la presencia de ésta, todo basado en los trabajos de Borsini (1944, 1946), pero sin tomar en cuenta los posteriores trabajos de Borsini (1966) y de Marticorena y Quezada (1985), en los cuales no se confirma la presencia de $V$. moyanoi para el territorio chileno. Al revisar el trabajo de Borsini (1944) se comprobó que la información entregada para la distribución geográfica de $V$. moyanoi corresponde a un mapa de distribución para varias especies del género, y que para el caso de V. moyanoi se extiende por todo el territorio argentino entre los paralelos $41^{\circ}$ y $50^{\circ}$ aproximadamente, pero sin entrar en el territorio chileno, además el material estudiado sólo corresponde a las provincias de Río Negro y Chubut, pero nada proveniente del territorio chileno. En tanto en el trabajo de Borsini (1946) la informa- 
ción entregada corresponde al mismo mapa de Borsini (1944), más nuevos materiales consultados, provenientes de las provincias argentinas de Chubut y Santa Cruz.

El hallazgo de V. moyanoi constituye un nuevo registro para la flora de Chile, y amplía el rango de distribución conocida para la especie en aproximadamente $46 \mathrm{~km}$ hacia el oeste, encontrándose protegida en la amplia zona de altas cumbres de la RNLJ. Con este registro el género Valeriana queda representado en Chile por 45 especies, y la familia Valerianaceae por 49.
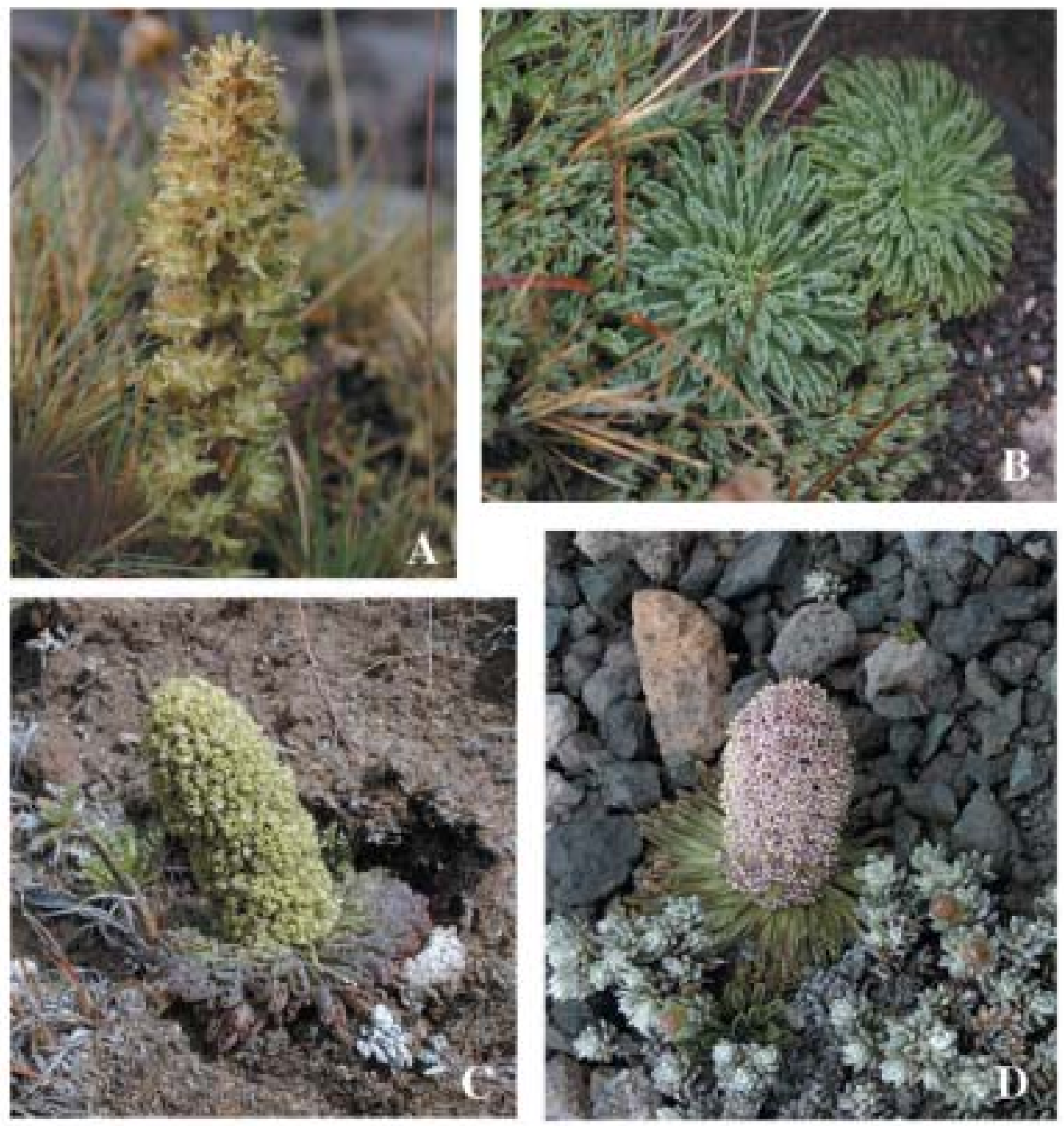

Figura 2. Valeriana moyanoi: A. con frutos, B. vegetativa, C y D. con flores (Fotografías A-B de P. Azócar, C-D de P. Saldivia).

Figure 2. Valeriana moyanoi: A. with fruits, B. vegetative, C \& D. with flowers (Photographers: A-B, P. Azócar; C-D, P. Saldivia). 
Gayana Bot. 63(2), 2006

TABLA I. Flora asociada a Valeriana moyanoi en Reserva Nacional Lago Jeinimeni y Cerro Rosado.

TABLE I. Associated flora to Valeriana moyanoi in Jeinimeni Lake National Reserve and Cerro Rosado.

\begin{tabular}{|c|c|c|c|}
\hline Familia & Especie & $\mathrm{Fv}^{*}$ & Lugar** \\
\hline \multirow[t]{5}{*}{ Asteraceae } & Perezia pedicularifolia Less. & $\mathrm{Hc}$ & RNLJ, CR \\
\hline & Perezia pilifera (D. Don) Hook. et Arn. & $\mathrm{Hc}$ & RNLJ \\
\hline & Senecio kingii Hook. f. & $\mathrm{Hc}$ & RNLJ \\
\hline & Senecio skottsbergii Cabrera & $\mathrm{C}$ & CR \\
\hline & Senecio subdiscoideus Sch. Bip. ex Wedd. & $\mathrm{Hc}$ & CR \\
\hline Brassicaceae & Menonvillea nordenskjoeldii (Dusén) Rollins & $\mathrm{Hc}$ & $\mathrm{CR}$ \\
\hline Caryophyllaceae & Silene sp. & $\mathrm{Hc}$ & CR \\
\hline Empetraceae & Empetrum rubrum Vahl ex Willd. & $\mathrm{C}$ & RNLJ \\
\hline Ericaceae & Gaultheria pumila ( L.f. ) D.J. Middleton & $\mathrm{C}$ & $\mathrm{CR}$ \\
\hline Iridaceae & Sisyrinchium sp. & $\mathrm{G}$ & CR \\
\hline Juncaceae & Luzula sp. & $\mathrm{Hc}$ & RNLJ, CR \\
\hline Ophiglossaceae & Botrychium dusenii (Christ) Alston & $\mathrm{Hc}$ & RNLJ \\
\hline Oxalidaceae & Oxalis loricata Dusén. & $\mathrm{G}$ & CR \\
\hline Ranunculaceae & Hamadryas kingii Hook. f. & $\mathrm{Hc}$ & RNLJ \\
\hline Rubiaceae & Oreopolus glacialis (Poepp. et Endl.) Ricardi & $\mathrm{C}$ & CR \\
\hline Scrophulariaceae & Calceolaria uniflora Lam. & $\mathrm{Hc}$ & RNLJ \\
\hline Valerianaceae & Valeriana fonkii Phil. & $\mathrm{Hc}$ & RNLJ \\
\hline
\end{tabular}

*: Fv = Forma de vida, $\mathrm{Hc}=$ Hemicriptófito, $\mathrm{C}=$ Caméfito, $\mathrm{G}=$ Geófito.

$* *: \mathrm{RNLJ}=$ Reserva Nacional Lago Jeinimeni, $\mathrm{CR}=$ Cerro Rosado.

\section{AGRADECIMIENTOS}

Los autores agradecen a Benjamín Molina de la RNLJ. A las señoras Inés Meza y Mélica Muñoz por su buena disposición para consultar el herbario (SGO) y para consultas bibliográficas. A Gustavo Saldivia por la confección de la Figura $\mathrm{N}^{\circ} 1$ y a Marcelo Rosas y Luis Faúndez por la revisión y aportes al presente trabajo.

\section{BIBLIOGRAFIA}

BorsinI, O. 1944. Valerianaceae. In: Descole, Genera et Species Plantarum Argentinarum 2: 275-372, tab. $132-160$.

Borsini, O. 1946. Addenda a las Valerianáceas argentinas II. Nuevos materiales consultados. Lilloa 12: 6785.

Borsini, O. 1966. Valerianáceas de Chile. Lilloa 32: 375476.

Borsini, O., R. Rossow \& M. Correa. 1999. Valerianaceae. En: Correa, M. N. (ed.), Flora Patagónica, parte VI. Colecciones Científicas del INTA. VIII. Buenos Aires, Argentina. 536 pp.

Gajardo, R. 1994. La vegetación natural de Chile. Cla- sificación y distribución geográfica. Editorial Universitaria. Santiago, $165 \mathrm{pp}$.

Henríquez, J., E. Pisano \& C. Marticorena. 1995. Catálogo de la flora vascular de Magallanes $\left(\mathrm{XII}^{\circ} \mathrm{Re}-\right.$ gión), Chile. Anales Instituto de la Patagonia, Serie Ciencias Naturales, Punta Arenas (Chile) 23: 5-30.

Marticorena, C. 1990. Contribución a la estadística de la flora vascular de Chile. Gayana Botánica 47: 85-113.

Marticorena, C. \& Quezada, M. 1985. Catálogo de la flora vascular de Chile. Gayana Botánica 42: 157.

Matthei, O. \& Espinoza, N. 1997. Valerianella rimosa Bastard (Valerianiaceae), Weed from the south of Chile. Gayana Botánica 54 (1):89-90.

Pisano, E. 1981. Bosquejo fitogeográfico de fuegopatagonia. Anales Instituto de la Patagonia, Punta Arenas (Chile) 12: 159-171.

Reese-Krug, H. \& F. Weberling. 1996. Zur Taxonomie hochandiner Valeriana - Arten (Valerianaceae). II. Valeriana globularis A.Gray, $V$. condamoana Graebn, und V. petersenii sp. nov. Botanische Jahrbuger. Systematik. 118 (2): 159-175.

XIFREDA, C. 1996. Valerianaceae. En: Zuloaga, F.O. y O. Morrone (eds.). Catálogo de las plantas vasculares de la República Argentina II, Dicotyledoneae. Monograph of Systematic Botany from the Missouri Botanical Garden 74:1130-1136.

Recibido: 28.02.06

Aceptado: 27.07.06 